\title{
Perspectivas e Direcionamentos de Algumas Pesquisas Apresentadas na Terceira Edição da Conferência Internacional em Pesquisa e Desenvolvimento de Livros Didáticos de Matemática
}

\section{Perspectives and Directions of Some Research Presented at the Third International Conference on Mathematics Textbook Research and Development}

\author{
Douglas Ribeiro Guimarães ${ }^{\mathrm{a}}$; Ana Paula Perovano ${ }^{\mathrm{ab}}$ \\ ${ }^{a}$ Universidade Estadual Paulista, Programa de Pós-Graduação Stricto Sensu em Educação Matemática. SP, Brasil

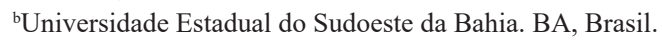 \\ *E-mail: apperovano@uesb.edu.br
}

\begin{abstract}
Resumo
O livro didático como objeto de estudo tem despertado o interesse de pesquisadores na Educação Matemática e, como resultado disso, eventos específicos ou grupos temáticos foram desenvolvidos para atender a essa crescente demanda. Assim, identificar quais focos temáticos sobre livros didáticos de matemática têm emergido nos estudos apresentados na terceira edição da Conferência Internacional em Pesquisa e Desenvolvimento de Livros Didáticos de Matemática foi o objetivo que direcionou nossa investigação. Adotamos uma abordagem qualitativa de pesquisa numa visão interpretativa dos dados e empregamos um mapeamento nos trabalhos publicados na referida conferência. Identificamos quatro categorias temáticas: análise do livro, livro digital, uso do livro e outros, contudo, neste texto, a discussão é destinada às três últimas temáticas. Os dados obtidos nos permitiram ter um panorama de como esse campo de pesquisa vem se desenvolvendo em várias partes do mundo. Diferentes perspectivas teóricas foram apresentadas na conferência e destacamos duas que foram recorrentes: a ideia do tetraedro sociodidático e a Capacidade de Design Pedagógico. No que tange às abordagens metodológicas, é predominante o paradigma da pesquisa qualitativa com o uso de vídeos, entrevistas, observação de aulas, mapeamento, análise de conteúdo e/ou a combinação de alguns desses métodos. Do que levantamos, identificamos que o campo de pesquisas sobre livros didáticos de matemática está evoluindo, ampliando as vertentes teóricas e consolidando alguns dos métodos empregados para análise e produção dos dados. Esperamos contribuir, no sentido de trazer mais elementos que possibilitem a compreensão da produção de conhecimento sobre o livro didático de matemática.
\end{abstract}

Palavras-chave: ICMT. Mapeamento. Uso do Livro Didático. Livro Digital.

\begin{abstract}
The textbook as an object of study has aroused the interest of researchers in Mathematics Education and, as a result, specific events or thematic groups have been developed to meet this growing demand. Thus, identifying which thematic focuses on mathematics textbooks have emerged in the studies presented in the Third International Conference on Mathematics Textbook Research and Development was the objective that guided our investigation. We adopt a qualitative approach to research in an interpretative view of the data and employ a mapping in the studies published at the referred conference. We identified four thematic categories: textbook analysis, digital textbook, textbook use and others, however, in this article, the focus is given to the last three themes. The data obtained allowed us to have an overview of how this field of research has been developing in various parts of the world. Different theoretical perspectives were presented at the conference and we highlight two that were recurrent: the idea of the socio-didactic tetrahedron and the Pedagogical Design Capacity. With regard to methodological approaches, the paradigm of qualitative research is predominant with the use of videos, interviews, class observation, mapping, content analysis and/or the combination of some of these methods. From what we have found, we identified that the field of research on mathematics textbooks is evolving, expanding the theoretical aspects and consolidating some of the methods used for data analysis and production. We hope to contribute towards bringing more elements that enable the understanding of the production of knowledge about the mathematics textbook.
\end{abstract}

Keywords: ICMT. Mapping. Textbook use; Digital Textbook.

\section{Introdução}

$\mathrm{O}$ ensino de matemática possui uma trajetória histórica atrelada aos livros didáticos (Valente, 2008), pois eles “desempenham um papel essencial na definição da matemática como uma disciplina, moldando as oportunidades de aprendizagem para os alunos e apoiando o trabalho, aprendizado e desenvolvimento profissional dos professores." (Rezat, Fan, Hattermann, Schumacher \& Wuschke, 2019, p. 3 , tradução nossa).

Recentemente, o interesse em investigar o livro didático tem se intensificado e as pesquisas realizadas buscam ampliar a compreensão desse recurso, de seu desenvolvimento e de como são atrelados à prática docente, ampliando a produção de conhecimento na área da Educação Matemática. Em decorrência disso, pesquisadores uniram-se em grupos de estudos ou em eventos específicos, como foi o caso do $13^{\circ}$ Congresso Internacional de Educação Matemática (ICME13), realizado na Alemanha, em 2016, que teve um Grupo de Estudo de Tópicos dedicado a recursos de aprendizagem, incluindo os livros didáticos. A Conferência Internacional em Pesquisa e Desenvolvimento de Livros Didáticos de 
Matemática $^{1}$ (ICMT) e a Conferência Internacional Recursos 2018 (Gitirana, Miyakawa, Rafalska, Soury-Lavergne \& Trouche, 2018) são mais dois exemplos. Listamos, aqui, apenas alguns dos vários eventos que estão discutindo o livro didático e temos o entendimento de que essa lista pode ser ampliada.

Nossa atenção está voltada para os livros didáticos de matemática. Assim, discorreremos brevemente sobre a ICMT. Sua primeira edição aconteceu em 2014, em Southampton, no Reino Unido, e a segunda em 2017, no Brasil, na cidade do Rio de Janeiro. A terceira edição da ICMT ocorreu na cidade de Paderborn, na Alemanha, no ano de 2019 e contou com quatro plenárias, quatro simpósios, dois workshops, 40 comunicações orais e 13 pôsteres, estes dois últimos, objetos de análise nesta pesquisa. Os trabalhos são fruto de pesquisadores de todos os continentes, o que mostra a relevância e riqueza a respeito das pesquisas com livro didático (Rezat et al., 2019). A quarta edição está prevista para acontecer em 2021, em Pequim, na China.

Compreender a produção de conhecimento sobre o livro didático de matemática é o objetivo de uma ampla pesquisa que estamos desenvolvendo e, neste texto, nosso foco de atenção busca responder quais focos temáticos sobre livros didáticos de matemática têm emergido nos estudos apresentados na terceira edição da Conferência Internacional em Pesquisa e Desenvolvimento de Livros Didáticos de Matemática (ICMT 3). Evidenciando, também, que aspectos e dimensões vêm sendo destacados e privilegiados, quais tendências teóricas e vertentes metodológicas foram empregadas nas pesquisas apresentadas na referida conferência.

\section{Desenvolvimento}

Para responder a pergunta diretriz, tivemos que fazer escolhas metodológicas, assim, adotamos uma abordagem qualitativa de pesquisa numa visão interpretativa dos dados produzidos, ou seja, lançamos nosso olhar para os dados, considerando que o tema é "organizado em torno da identificação do contexto social, político ou histórico do problema que está sendo investigado." (Creswell, 2010, p. 209). Dessa forma, apresentamos a interpretação que obtivemos ao identificar os focos temáticos sobre livros didáticos de matemática na ICMT 3.

$\mathrm{O}$ procedimento metodológico que utilizamos foi $\mathrm{o}$ mapeamento da pesquisa que é, segundo Fiorentini et al. (2016, p. 18), "um processo sistemático de levantamento e descrição de informações acerca das pesquisas produzidas sobre um campo específico de estudo, abrangendo um determinado espaço (lugar) e período de tempo." Segundo os autores citados, as informações referem-se aos aspectos de produção, como a descrição do local e da quantidade de estudos, a identificação dos autores e dos participantes das pesquisas; além desses, fazem parte desse mapeamento informações sobre os aspectos teóricos e metodológicos e quais temas surgiram nessa busca.

Dessa forma, exibiremos um "quadro holístico", que é a apresentação do modelo que "envolve o relato de múltiplas perspectivas, a identificação dos muitos fatores envolvidos em uma situação e, em geral, o esboço do quadro mais amplo que emerge." (Creswell, 2010, p. 210), que pode ajudar os pesquisadores a se orientar mais facilmente nas áreas problemáticas. $\mathrm{Na}$ próxima subseção, trataremos dos procedimentos metodológicos de busca e seleção das publicações empregadas em nossa análise.

\subsection{Os procedimentos de busca e seleção dos textos utilizados no mapeamento}

Neste recorte da pesquisa, o objeto de estudo são os anais. Faziam parte dos anais os textos das plenárias, simpósios, workshops, comunicações e pôsteres. Selecionamos, para nossa análise, as publicações em formato de comunicação e pôster, pois eram textos mais “completos". Já as pesquisas das plenárias, simpósios e workshops continham as ideias iniciais sobre o que iria ocorrer no dia do evento; como os trabalhos são publicados antes da conferência, é possível a ocorrência de alterações nas referidas modalidades.

Iniciamos a leitura dos anais pelos títulos e pelos resumos. O resumo tem como finalidade divulgar os trabalhos produzidos na esfera acadêmica, devendo conter os elementos essenciais da pesquisa, como o que se deseja investigar, a trajetória metodológica e uma discussão dos resultados alcançados (Ferreira, 2002).

Borba, Almeida e Gracias (2018) apontam que cinco informações precisam constar nos resumos: pergunta de pesquisa ou objetivos, referenciais teóricos e metodológicos, dados da pesquisa e procedimentos, resultados da pesquisa e, por fim, as conclusões ou reflexões. Entretanto, "nem todo resumo traz em si mesmo e de idêntica maneira todas as convenções previstas pelo gênero: em alguns, falta a conclusão da pesquisa; em outros, falta o percurso metodológico, ainda em outros, pode ser encontrado um estilo mais narrativo." (Ferreira, 2002, p. 268). Assim, ler o resumo pode não trazer todos os elementos de que necessitamos sobre o texto, de modo a categorizá-lo corretamente.

Em decorrência disso, realizamos um movimento oscilatório de idas e vindas ao resumo e ao texto completo, de modo a obter elementos que o caracterizassem, fornecendo indícios para a análise. Nesse mesmo sentido, para uma melhor compreensão, "cada resumo deve ser lido e analisado numa relação de dependência com o trabalho na íntegra, mas também enquanto realidade relativamente independente." (Ferreira, 2002, p. 270).

Dessa forma, todos os textos das 40 comunicações e 
dos 13 pôsteres foram lidos integralmente e classificados individualmente. Cabe mencionar que, para validação da classificação, cada autor avaliou a rotulação do outro e, em caso de dúvida, a categorização foi discutida até entrar em consenso.

Emergiram, assim, categorias e subcategorias a respeito dos focos temáticos das pesquisas sobre livros didáticos de matemática: análise do livro (pelos pesquisadores ou por outros sujeitos, além de análise comparativa e de conteúdo), livro digital, uso do livro (pelo aluno, pelo professor, ou por ambos) e, outros (abordando temas como inclusão, marketing, elaboração de livro didático e revisão de literatura). A categoria análise do livro é a mais pesquisada e discutida na Educação Matemática (Fan, Zhu \& Miao, 2013), o que foi evidenciado também neste mapeamento, visto que 30 dos 53 estudos se enquadravam nesta vertente. As outras três categorias são inovadoras, teórica e metodologicamente, apresentando novas perspectivas para a pesquisa sobre livros didáticos, dessa forma, nossa opção é por apresentar essas categorias. Outrossim, entendemos que a análise do livro continua sendo uma perspectiva importante, visto o impacto desse recurso na prática pedagógica e nas relações curriculares, assim, apresentaremos os dados referentes a essa categoria em outra publicação.

\subsection{Resumos dos Trabalhos}

Conforme pontuamos anteriormente, trataremos de três das quatro categorias que emergiram a partir de nossas leituras, quais sejam: livro digital, uso do livro e outros. Passaremos a descrevê-los e a incluir, brevemente, uma visão geral de cada categoria, para posterior aprofundamento em seus aspectos teóricos e metodológicos. As categorias também podem ser vistas no Quadro 1.

Quadro 1 - Distribuição das categorias, seus respectivos autores, ideias principais e métodos de pesquisa

\begin{tabular}{|c|c|c|c|c|}
\hline \multicolumn{2}{|c|}{ Categoria } & Autores & Ideias e/ou Referencial Teórico & Método \\
\hline \multirow{4}{*}{\multicolumn{2}{|c|}{$\begin{array}{c}\text { Livro Digital } \\
\text { (4) } \\
\text { Brnic } \\
\text { Tanaka e Hayata } \\
\text { Goto }\end{array}$}} & Edson, Phillips e Bieda & $\begin{array}{l}\text { Engajamento disciplinar produtivo } \\
\text { (Engle \& Conant, 2002) }\end{array}$ & $\begin{array}{l}\text { Relato e mapeamento de } \\
\text { conjecturas }\end{array}$ \\
\hline & & $\begin{array}{l}\text { LD como tradutor de políticas e } \\
\text { elementos do LD digital }\end{array}$ & $\begin{array}{l}\text { Comparação entre grupos de } \\
\text { alunos e professores; utilização de } \\
\text { pré e pós testes. }\end{array}$ & \\
\hline & & $\begin{array}{l}\text { Teoria da Generalização de } \\
\text { Dörfler (1991) }\end{array}$ & $\begin{array}{l}\text { Comparação e análise de LD } \\
\text { digital e impresso }\end{array}$ & \\
\hline & & Sem explicitação & Estado da Arte & \\
\hline & \multirow{3}{*}{$\begin{array}{c}\text { pelo } \\
\text { professor (3) }\end{array}$} & Leshota & $\begin{array}{l}\text { Capacidade de Design Pedagógico } \\
\text { dos professores (Brown, 2009) }\end{array}$ & $\begin{array}{l}\text { Gravação da aula, fotos, } \\
\text { observação e entrevista pós- } \\
\text { observação. }\end{array}$ \\
\hline & & Mizoguchi e Shinno & $\begin{array}{l}\text { Teoria Antropológica do Didático } \\
\text { (noções de transposição e } \\
\text { praxeologia didática) }\end{array}$ & $\begin{array}{l}\text { Quadro para analisar o livro a } \\
\text { partir da teoria }\end{array}$ \\
\hline & & Schorcht & $\begin{array}{l}\text { Capacidade de Design Pedagógico } \\
\text { dos professores (Brown, 2009) }\end{array}$ & $\begin{array}{l}\text { Grade do repertório de Kelly } \\
\text { (1955) como uma ferramenta de } \\
\text { avaliação psicológica, pré e pós- } \\
\text { entrevista online durante o curso }\end{array}$ \\
\hline & \multirow{4}{*}{$\begin{array}{l}\text { pelo aluno } \\
\quad(4)\end{array}$} & De Almeida e Silva & $\begin{array}{l}\text { Semiótica de Pierce (raciocínio } \\
\text { diagramático) }\end{array}$ & $\begin{array}{l}\text { Leitura do texto do LD e resolução } \\
\text { de uma atividade sem a utilização } \\
\text { do livro }\end{array}$ \\
\hline & & Sievert, Van den Ham e Heinze & $\begin{array}{l}\text { Princípios aritméticos (Kilpatrick } \\
\text { et al., 2001) } \\
\text { Desenvolvimento da competência } \\
\text { estratégica (Lemaire \& Siegler, } \\
\text { 1995) }\end{array}$ & $\begin{array}{l}\text { Análise do livro e do desempenho } \\
\text { dos alunos }\end{array}$ \\
\hline & & $\begin{array}{l}\text { Slisko, Hernández Rebollar, } \\
\text { Nabor Sánchez e Ramirez Herrera }\end{array}$ & $\begin{array}{l}\text { Apresenta um levantamento da } \\
\text { literatura sobre livro didático }\end{array}$ & $\begin{array}{l}\text { Análise das respostas dos alunos } \\
\text { de duas tarefas retiradas do livro } \\
\text { (uma parte do livro que os alunos } \\
\text { já conheciam e outra da parte } \\
\text { desconhecida) }\end{array}$ \\
\hline & & Mesa, Liakos e Zhang & $\begin{array}{l}\text { Tetraedro Sociodidático (Rezat \& } \\
\text { Sträßer, 2012) }\end{array}$ & $\begin{array}{l}\text { Identificação das competências } \\
\text { matemáticas oferecidas pelo livro, } \\
\text { codificação dos conteúdos do livro } \\
\text { aberto e análise das respostas da } \\
\text { pesquisa }\end{array}$ \\
\hline & $\begin{array}{l}\text { aluno e pelo } \\
\text { professor } \\
\text { (1) }\end{array}$ & Glasnović Gracin e Jukić Matić & $\begin{array}{l}\text { Tetraedro Sociodidático (Rezat \& } \\
\text { Sträßer, 2012) }\end{array}$ & $\begin{array}{l}\text { Estudo de caso, observação em } \\
\text { sala de aula ( } 4 \text { aulas) e entrevista }\end{array}$ \\
\hline
\end{tabular}


Continuação

\begin{tabular}{|c|c|c|c|}
\hline Categoria & Autores & Ideias e/ou Referencial Teórico & Método \\
\hline \multirow{8}{*}{$\begin{array}{c}\text { Outros } \\
(\mathbf{8}) \\
\text { Højgaard } \\
\text { Mabuchi e Hayata } \\
\text { Häsel-Weide e } \\
\text { Nührenbörger } \\
\text { Shen, Bao e Zhang } \\
\text { Janke } \\
\text { Tarp } \\
\text { Watanabe }\end{array}$} & Andrade-Molina e Montecino & $\begin{array}{l}\text { Estratégia analítica rizomática } \\
\text { (Deleuze \& Guattari, 1987). } \\
\text { Ideias do Neoliberalismo }\end{array}$ & Pesquisa exploratória \\
\hline & $\begin{array}{l}\text { Design de livros didáticos } \\
\text { Competências matemáticas } \\
\text { apresentadas e exemplificadas no } \\
\text { relatório KOM (Niss \& Højgaard, } \\
\text { no prelo) } \\
\text { Modelo tridimensional de } \\
\text { competências } \times \text { conceitos } \times \text { nota } \\
\text { para decidir sobre o conteúdo e } \\
\text { os objetivos das várias partes dos } \\
\text { livros da série Matematrix }\end{array}$ & $\begin{array}{l}\text { Descritiva com apresentação de } \\
\text { como foi feito o workshop }\end{array}$ & \\
\hline & $\begin{array}{l}\text { Termo "paradigma” de Kuhn } \\
\text { (1970) } \\
\text { e "programa de pesquisa" de } \\
\text { Lakatos (1978) } \\
\text { Construção própria da estrutura } \\
\text { 'programa de pesquisa } \\
\text { educacional' }\end{array}$ & $\begin{array}{l}\text { Consideração filosófica para o } \\
\text { livro didático de matemática, } \\
\text { porque reflete as condições e } \\
\text { restrições sociais e ideológicas da } \\
\text { era da modernização }\end{array}$ & \\
\hline & $\begin{array}{l}\text { Inclusão (Scherer et al., 2016) } \\
\text { Inclusão e matemática (Häsel- } \\
\text { Weide \& Nührenbörger, 2017) } \\
\text { Design Science (Nührenbörger et } \\
\text { al., 2016) }\end{array}$ & $\begin{array}{l}\text { Lições videografadas, } \\
\text { considerando as ideias de } \\
\text { sensibilidade das diferenças, } \\
\text { linguagem e materiais e conexões } \\
\text { de aprendizagem social e } \\
\text { relacionada ao conteúdo. } \\
\end{array}$ & \\
\hline & $\begin{array}{l}\text { Conceito de representação (De } \\
\text { Bock, Van Dooren \& Verschaffel, } \\
\text { 2015) }\end{array}$ & Meta-análise e análise de conteúdo & \\
\hline & Sem explicitação & $\begin{array}{l}\text { Apresentação de como foi } \\
\text { desenvolvido o workshop } \\
\text { desenvolvido pelo autor sobre } \\
\text { ensinar a escrever livros didáticos } \\
\text { de matemática }\end{array}$ & \\
\hline & $\begin{array}{l}\text { Abordagem funcional da } \\
\text { linguagem (Halliday, 1973) } \\
\text { Competências para dominar } \\
\text { muitos, contando e adicionando } \\
\text { (Tarp, 2018) }\end{array}$ & Pesquisa Teórica & \\
\hline & $\begin{array}{l}\text { Conceito de habilidade espacial. } \\
\text { Modelo teórico (Newcombe \& } \\
\text { Shipley, 2015; Uttal et al., 2013). }\end{array}$ & Lesson Study (estudo de aula) & \\
\hline
\end{tabular}

Fonte: Dados da pesquisa.

\subsubsection{Livro digital}

Encontramos definições diferentes para livros digitais na literatura. Naftaliev (2018), por exemplo, explica que os livros eletrônicos ou livros interativos, são diferentes dos livros estáticos por exigirem uma maior interatividade do usuário; dessa forma, são mais complicados de se trabalhar e incluem ferramentas de manipulação, construção e ativação de exemplos. Já Pepin, Gueudet, Yerushalmy, Trouche e Chazan (2015) definem os livros eletrônicos em três modelos: dinâmicos, em que um livro estático é vinculado com outros objetos de aprendizagem; "vivos" ou em evolução, em que os livros são criados de maneira cumulativa e dinâmica; e os interativos, baseados em um modelo composto por ferramentas e em que as tarefas e os materiais interativos podem ser vinculados e combinados de diferentes maneiras. Destacamos que, na visão desses autores, os livros estáticos podem ser impressos ou digitais, uma vez considerando “digitais" uma cópia do livro impresso, sem modificações.

Quatro pesquisas relacionadas ao livro digital foram encontradas na ICMT 3 e apresentam similaridades com as ideias de Naftaliev (2018) e de Pepin et al. (2015), e que, apesar de não se referirem aos autores citados, mostram que os livros digitais promovem interações entre os estudantes. Edson, Phillips e Bieda (2019), por exemplo, descrevem os esforços para a transição de um currículo impresso para o digital nos Estados Unidos. Os autores se apoiam em princípios do envolvimento disciplinar produtivo, que envolve problematização, autoridade, responsabilidade e recursos, de modo que os estudantes abordam problemas matemáticos, compartilham com os colegas seus pensamentos e adotam ideias matemáticas para comunicar e revisar o trabalho feito, tudo isso se apoiando em recursos como tempo, local e tecnologias.

O ambiente virtual é, então, composto de modo que os 
alunos possam interagir uns com os outros e com o professor; os autores explicam que essa dinâmica é diferente da sala de aula física, pois os estudantes estão em contato com todos os alunos, num ambiente que oportuniza as discussões em tempo real, na tela do computador de cada um. Assim, Edson et al. (2019) entendem que, tanto a matemática quanto as relações sociais são aprimoradas ao fazer uso desse ambiente virtual colaborativo.

Brnic (2019), ao comparar os livros impressos e digitais alemães, mostra as diferenças entre eles na estrutura e na exposição de seus elementos dinâmicos. O autor compreende que o livro didático é um tradutor de políticas públicas e que a estrutura dele pode afetar a aprendizagem dos alunos. Brnic (2019) tem por objetivo entender o desempenho matemático dos alunos, além de suas crenças, quando utilizam um livro digital. Assim, o autor adota procedimentos metodológicos com dois grupos diferentes: um, que fará uso do livro impresso e outro com os livros digitais, além do que, serão aplicados testes, antes e depois dessa experiência, para então analisar os conhecimentos dos estudantes e uma consequente comparação entre os grupos. $\mathrm{O}$ autor apresentou os resultados no dia do evento, o que nos impediu o acesso às suas conclusões.

Tanaka e Hayata (2019), por sua vez, apresentam as habilidades ou noções algébricas presentes em um livro digital japonês do Ensino Médio. Os autores compreendem que no aprendizado de Álgebra, a generalização desempenha um papel essencial e, desse modo, baseiam-se na teoria de generalização de Dörfler (1991), em “que os alunos abstraem esses relacionamentos [entre objetos matemáticos] por meio de suas próprias atividades." (Tanaka \& Hayata, 2019, p. 387). Entre os tópicos possíveis para fazer a análise, os autores optaram pelo estudo da função do $2^{\circ}$ grau e encontraram que as ferramentas digitais para esse conteúdo fornecem apenas validações ou justificações sobre as sentenças e, assim, não conseguem estabelecer o que Dörfler defende. Desse modo, Tanaka e Hayata (2019) pretendem analisar outros conteúdos de livros digitais de outros países, e compreender quais ferramentas digitais conseguiriam desenvolver as atividades dos estudantes, para que estabeleçam relações entre os objetos matemáticos.

Por fim, Goto (2019) explica o estado atual dos materiais digitais, também no Japão, afirmando que esses materiais são amplamente utilizados no país. O autor afirma que eles são diferentes dos livros didáticos digitalizados, uma vez que apresentam dois objetivos: complementação do livro didático e conteúdos livres para desenvolver as habilidades matemáticas que não estão presentes nos conteúdos dos livros. O estado atual pode ser resumido em quatro conteúdos: design da lição ou da aula, a habilidade desenvolvida ao usar o material, o momento em que deve ser utilizado e a distinção entre software comercial e materiais didáticos criados por eles mesmos. Goto (2019) argumenta que os estudos para construir esse estado atual foram mistos, sendo necessário aprofundamento em cada um dos quatro conteúdos.

Entendemos que tais trabalhos indicam caminhos promissores para a pesquisa com livros digitais, pois mostram que os estudantes podem atuar em colaboração, em ambientes dinâmicos, além de oportunizar habilidades que os livros impressos não conseguem oferecer. Apesar de as pesquisas versarem sobre o livro didático digital, a metodologia utilizada nos trabalhos foi distinta, sendo: mapeamento e estado da arte, comparação e análise do livro e comparação entre grupos de alunos.

\subsubsection{Uso do livro didático}

Fan et al. (2013) argumentam que é importante examinar os livros didáticos não apenas em termos de seu conteúdo e estrutura, mas também de seu uso em salas de aula reais, contemplando seus dois usuários: o aluno e o professor. $\mathrm{Na}$ terceira conferência, identificamos oito publicações que versavam sobre esse tema, tratando dos dois usuários, individual ou coletivamente.

O uso do livro pelo professor pode nos fornecer um indício sobre como os livros didáticos direcionam a maneira de ensinar matemática. Na ICMT 3 foram identificadas três pesquisas relacionadas a essa temática. Leshota (2019) explora as possibilidades e restrições de um plano de aula com scripts e como um professor os mobiliza. Implementados em 2016, numa província da África do Sul, em escolas com baixo desempenho, os scripts são planos de aula expandidos que, além de especificar o conteúdo, apresentam o sequenciamento e a duração de cada componente da aula. A aula de uma professora foi gravada e fotografada com intuito de observar se as explicações instrucionais eram fornecidas ou não e a mobilização (ou não) por parte da professora, à medida que ensinava o conteúdo de fatoração.

Após alguns meses, foi realizada uma entrevista com a professora, com a intenção de verificar as interações dela com os scripts. Em seus resultados, a autora relata que a Capacidade de Design Pedagógico da professora investigada, naquela tarefa observada, não é alta e é desigual e aponta alguns desafios no que tange a pesquisas, cujos contextos possuam alta prescrição: a não percepção dos recursos e restrições do script, a possibilidade de desviar-se do script e omitir certos aspectos críticos ao implementá-lo. Os resultados sugerem que as interações dos professores com os scripts que podem ser tácitas e não totalmente participativas e são semelhantes no estudo com livros didáticos.

Mizoguchi e Shinno (2019) buscam caracterizar o kyozai-kenkyu dos professores primários japoneses ao usar livros didáticos de matemática. "Literalmente, a palavra kyozai significa material didático ou assunto, e kenkyu se refere à pesquisa ou ao estudo. Em uma prática de kyozaikenkyu, o design de tarefas está envolvido e os livros didáticos são frequentemente usados como um recurso principal.” (Mizoguchi \& Shinno, 2019, p. 257). Para 
efetivar a caracterização, os autores fundamentam-se na Teoria Antropológica do Didático e empregam as noções de transposição e praxeologia didática. Os autores analisaram o livro didático utilizado por dois professores em suas aulas (adição de frações com mesmo denominador e comparação de frações) e identificaram que os professores modificaram as lições apresentadas no livro na esperança de melhorar o aprendizado de seus alunos com abordagens diferentes (enquanto o livro didático visa simplesmente pensar em como adicionar frações, o que o professor pretendia era que os alunos entendessem por que a maneira que ele apresentava no quadro era certa; o objetivo do professor na lição é um novo objetivo matemático além do apresentado no livro didático). Os autores evidenciaram que "os tipos de kyozai-kenkyu nem sempre aparecem com base nas habilidades profissionais ou matemáticas dos professores; pelo contrário, o tipo depende da comunidade ou da situação em que o professor está realmente envolvido." (Mizoguchi \& Shinno, 2019, p. 261).

Identificar a linguagem dos professores sobre tarefas relacionadas à história da matemática é o objetivo da pesquisa desenvolvida por Schorcht (2019). De acordo com o autor, para projetar suas lições, os professores usam recursos e o conceito de Capacidade de Design Pedagógico, que ajuda a entender a interação entre professores e esses recursos. Foram empregadas entrevistas online antes e após a situação apresentada aos sujeitos de pesquisa.

$\mathrm{Na}$ entrevista que antecedeu a situação, foi utilizada a metodologia da grade do repertório (Kelly, 1955) como uma ferramenta de avaliação psicológica em que as pessoas entrevistadas geram uma lista de possíveis itens de interesse. Depois disso, o entrevistador mostra às pessoas do teste três itens de sua lista. As pessoas devem nomear a semelhança de dois itens e descrever o terceiro de uma maneira diferente. Esses pares de atributos contêm uma característica e seu oposto, e isso fornece uma visão perspicaz das crenças em relação ao contexto. Os resultados evidenciam visões diferentes sobre trechos históricos que podem ser relacionados à aparência das tarefas, ao conteúdo, a algumas observações matemáticas e relacionadas ao uso em sala de aula. O autor evidencia um uso incompreendido de tarefas sobre história da matemática nas salas de aula, possivelmente pela existência de uma lacuna entre a interpretação dos professores e as reivindicações dos pesquisadores.

Quatro pesquisas foram enquadradas como sendo referentes ao uso do livro didático pelo aluno. Estes estudos, segundo Rezat (2011), são raros, no entanto, nesta conferência, se mostra uma certa ampliação no número de estudos que tratam desta temática. De Almeida e Silva (2019) analisaram as inscrições dos alunos ao usar um livro de Cálculo Diferencial e Integral para aprender o conceito de integral dupla; para tanto, utilizaram o conceito de raciocínio diagramático de Charles Peirce, pois, por meio desse conceito, é possível elucidar o desenvolvimento do conhecimento em uma atividade através de três etapas: construir sinais, experimentar com eles e observar os resultados. Participaram como sujeitos 35 alunos de um curso de engenharia de uma universidade brasileira que efetuaram a leitura do livro do aluno e resolveram uma atividade que apresenta a introdução do conceito de integral dupla sem a utilização do livro (Cálculo, vol. 2, $7^{\mathrm{a}}$ edição, escrito por James Stewart, versão traduzida para o português).

As autoras evidenciam que a maneira como o livro introduz o conceito de integral dupla favorece o processo de aprendizagem dos alunos. O raciocínio diagramático dos alunos foi acionado por imagens, problemas e linguagem, conforme incluído no livro. Assim, a caracterização desse raciocínio pode fornecer um quadro teórico para descrever e interpretar a atividade de aprendizagem dos alunos e o uso de diagramas, no caso em questão, para aprender o conceito integral dupla.

Sievert, Van den Ham e Heinze (2019) apresentam três estudos que abordam efeitos dos livros didáticos sobre a aprendizagem dos alunos no campo da aritmética da escola primária, usando uma amostra longitudinal em larga escala de 1.664 alunos do $1^{\circ}$ ao $3^{\circ}$ ano. O primeiro estudo visa analisar os efeitos de diferentes livros didáticos que representam o mesmo currículo no desempenho dos alunos. O segundo e terceiro estudos aprofundam os resultados do estudo 1. Eles examinam a qualidade das oportunidades de aprender com esses livros sobre o uso de princípios aritméticos e a escolha de estratégias adaptativas e analisam o efeito da qualidade dos livros sobre o desempenho dos alunos nesses domínios.

Os autores empregaram critérios das quatro dimensões do modelo proposto por Lemaire e Siegler (1995) e aplicaram aos livros didáticos de Grau 1 (princípios aritméticos) e Grau 2 e 3 (escolha da estratégia adaptativa). O estudo 1 revelou diferenças significativas no desempenho aritmético dos alunos em todos os níveis da série. As análises do estudo 2 mostram efeitos substanciais da qualidade dos livros didáticos da primeira série, no que diz respeito às oportunidades de aprendizado para o uso de princípios aritméticos no uso real desses princípios pelos alunos, no final do primeiro ano de escolaridade. O estudo 3 mostrou um efeito significativo da qualidade dos livros didáticos sobre o desempenho dos alunos. Assim, os autores concluem que há efeitos dos livros didáticos no aprendizado dos alunos, ou seja, se os livros didáticos possuem diferentes níveis de qualidade institucional e essas diferenças influenciam o aprendizado dos alunos, devem ser fornecidos aos alunos os melhores livros para o seu aprendizado.

O estudo de Slisko, Hernández Rebollar, Nabor Sánchez e Ramirez Herrera (2019) busca descobrir o que os alunos mexicanos do Ensino Fundamental tiram de uma sequência de aprendizado inadequada (que inclui contexto artificial, perguntas pouco claras sobre conceitos e cálculos e tarefas complicadas, desnecessárias, de desenho) e de uma declaração errônea de fatos ("quatro de dez" é 37\%) no livro didático de matemática que usam. Os autores afirmam que há muito tempo existe uma preocupação com os erros dos alunos 
quando estão aprendendo matemática e pouca atenção foi dada a pesquisas envolvendo erros cometidos por autores dos livros didáticos de matemática, principalmente relacionados ao uso de "contextos problemáticos" inventados e irreais que criaram uma imagem inadequada de "aplicações matemáticas" na Educação (Korsunsky, 2002, Pollak, 1968).

Duas tarefas foram apresentadas a 143 alunos do $7^{\circ}$ ano, em que a primeira tarefa foi da parte do livro que os alunos conheciam e a segunda parte ainda desconhecida pelos alunos que tiveram 50 minutos para responder individualmente. Das respostas, os autores identificaram que as sequências inadequadas de aprendizado apresentadas em livros didáticos influenciam negativamente o desempenho dos alunos, tanto no nível processual quanto na coerência entre as representações visuais e numéricas. Dessa forma, cabe ao professor ajudar a evitar essas influências, caso os processos de revisão das editoras e Ministério da Educação não consigam eliminar essas sequências inadequadas. Uma sugestão seria, ao receber uma tarefa com sequência inadequada, solicitar que os alunos corrijam e reformulem o problema, o que proporciona uma oportunidade de prática de leitura e pensamento crítico para os alunos que entenderiam que os livros didáticos de matemática não são materiais livres de erros.

O tetraedro sociodidático (Rezat \& Sträßer, 2012) foi a fundamentação teórica utilizada por Mesa, Liakos e Zhang (2019) para analisar como o conteúdo matemático de um livro é absorvido pelos instrutores, ao planejar e ensinar suas lições, e pelos alunos que participam dessas lições. Foram analisadas as respostas de mais de 100 alunos que cursaram Álgebra Linear, com a versão HTML do livro didático Álgebra Linear (Beezer, 2019) que é um livro dinâmico de código aberto escrito usando o PreTeXt ${ }^{2}$ que captura a estrutura dos livros didáticos para facilitar a conversão para vários outros formatos, nesse caso, um livro interativo online. Foram analisadas as respostas dos alunos e os resultados indicaram uma associação entre o conteúdo matemático apresentado no livro didático e as competências que os alunos provavelmente serão solicitados a colocar em ação e, possivelmente, melhorar ao interagirem com os recursos específicos do livro, ou seja, as competências oferecidas pelos recursos do livro didático interagiram com em determinado grau com os alunos.

Mais raros ainda são os estudos que examinam como, coletivamente, professores e alunos usam esse recurso (Glasnović Gracin \& Jukić Matić, 2019) e, nesta conferência, essa tendência se mantém, visto que foi apresentado apenas um dos oito trabalhos categorizados nesta temática. Com o objetivo de investigar e contrastar a utilização de livros didáticos da Croácia vista, simultaneamente, por um professor e por seus alunos, Glasnović Gracin e Jukić Matić (2019) entendem que o conteúdo e a estrutura dos livros afetam as escolhas dos professores de diferentes maneiras, durante os processos de planejamento e execução de uma lição. Para examinar a utilização dos livros didáticos de matemática do ponto de vista do professor e dos alunos foi empregado o tetraedro sociodidático (TSD) de Rezat e Sträßer (2012) evidenciando quais conexões entre os vértices do TSD se tornam as mais proeminentes e as mais poderosas para os alunos e seus professores durante o processo de uso de um livro didático.

Foi realizado um estudo de caso que envolveu uma professora de matemática do Ensino Fundamental na Croácia (séries de cinco a oito) e seis de suas alunas, divididas em dois grupos. Foram usadas a observação de quatro aulas e entrevista semiestruturada, ficando evidenciado que o livro foi utilizado, em grande parte, para adquirir novos conteúdos, praticar e atribuir tarefas de casa. A representação da sala de aula e o uso do livro foram afetados pelas crenças e intenções do professor. Os resultados evidenciam que o uso do livro pelos alunos depende do processo de mediação do professor entre o aluno e o livro, e que muitas das decisões do professor sobre o uso do livro são influenciadas pelas normas dos componentes sociais sobre ser professor. Além do conteúdo, professores e alunos usam ou não usam um livro didático por motivos relacionados a instituições, convenções e normas de professor e aluno, e por outros motivos sociais e institucionais.

Dos três trabalhos que versavam sobre o uso do livro didático pelo professor, dois utilizam o conceito de Capacidade de Design Pedagógico para tratar da interação entre o livro didático e o professor, em tarefas que abordam um conteúdo matemático ou histórico matemático. A Didática da Matemática faz-se presente nessa categoria com a Teoria Antropológica do Didático empregada na pesquisa de Mizoguchi e Shinno (2019) e o tetraedro sociodidático empregado em duas pesquisas, uma pesquisa com foco no uso do livro didático pelos alunos e na única pesquisa com foco no uso do livro didático pelo professor e pelo aluno. A semiótica de Pierce foi empregada no trabalho de De Almeida e Silva (2019).

As metodologias adotadas são de abordagem qualitativa, em que foram utilizados os procedimentos de gravação da aula, fotos, entrevistas e análise das respostas dos alunos. Destacamos a pesquisa de Schorcht (2019) que empregou uma ferramenta de avaliação psicológica como possibilidade para fornecer uma visão das crenças dos professores, a respeito dos contextos apresentados em relação ao conteúdo histórico matemático.

\subsubsection{Outros}

São incluídas nessa categoria as pesquisas que não se encaixam nas anteriores e que apresentam poucos estudos para formar uma categoria nova. Entre os focos de estudo presentes na ICMT 3, destacamos os trabalhos que problematizam a

2 https://pretextbook.org/. 
distribuição dos livros didáticos e a escrita desses recursos, seja a partir do currículo, seja em projetos com professores; os olhares filosóficos sobre os livros e as relações com práticas inclusivas, além dos trabalhos do tipo meta-análise. Apesar disso, encontramos dois estudos que não realizam discussões sobre o livro didático em si ou a aspectos mais próximos desse recurso.

Andrade-Molina e Montecino (2019) têm como objetivo problematizar a distribuição e as estratégias de mercado dos manuais escolares de matemática em um contexto incorporado nas políticas e suposições neoliberais sobre Educação. Os autores exploram reportagens da mídia que abordaram a produção, distribuição e seleção dos livros didáticos e de como o mercado chileno atua nesse ramo. Andrade-Molina e Montecino (2019) mostram que há uma diferença nos livros que chegam a escolas públicas, que são distribuídos gratuitamente pelo governo, e os livros das escolas particulares, em que esse material deve ser comprado. Ademais, os autores veem essas características neoliberais como perigosas, por segregar estudantes em condições socioeconômicas distintas. Por fim, pretendem empregar um estudo de caso em uma escola chilena que aceitou ofertas de vendedores de livros e compreender a situação dos professores frente a essa demanda.

Apresentar um dos elementos-chave para o desenvolvimento de competências, que estão presentes em livros didáticos da Dinamarca, é o objetivo de Højgaard (2019). Esse elemento-chave é um modelo tridimensional de conteúdo e objetivos, que combina competências matemáticas, conceitos matemáticos principais e nível de escolaridade. Como o pesquisador também é um designer e autor de livros didáticos, ele descreve como os livros da coleção "Matematrix" atendem ao modelo proposto e, assim, esse modelo estrutura as discussões e as progressões em cada conceito, especificando os títulos e os objetivos de aprendizagem de cada capítulo do livro didático.

Mabuchi e Hayata (2019) têm como objetivo descrever uma ideologia da era da modernização na Educação Matemática usando o referencial teórico desenvolvido por eles. Esse referencial é construído a partir de uma discussão sobre paradigma e programa de pesquisa, chegando a uma estrutura, nomeada pelos autores de "programa de pesquisa educacional". O programa consiste de três níveis inseparáveis: nível racional, nível de crença e nível da sociedade. Segundo os autores, metodologicamente, eles fizeram uma consideração filosófica para o livro didático de matemática japonês, porque, para Mabuchi e Hayata (2019), ele reflete as condições e restrições sociais e ideológicas da era da modernização.

Como resultado, perceberam que a modernização mudou o conteúdo e a disposição do conhecimento matemático no currículo, atacando, assim, os níveis de crença e da sociedade, não modificando o nível racional. Desse modo, concluem que outras investigações são necessárias para compreender os motivos dessas modificações nos dois níveis citados e a não modificação no nível racional.
Häsel-Weide e Nührenbörger (2019) têm como objetivo identificar práticas inclusivas nas aulas de matemática de escolas primárias inclusivas. Os autores analisam um livro didático alemão, considerando-o como artefato que medeia a atividade em sala de aula e, ainda, abordam um exemplo em que alunos e professores estão resolvendo atividades de subtração que foram baseadas nesse livro. O projeto em que ocorrem essas atividades está baseado no paradigma de design science (Nührenbörger et al., 2016), além de a análise seguir uma perspectiva interacionista, com foco na microcultura da sala de aula e nas práticas matemáticas. As atividades são gravadas e, posteriormente, analisadas de diversas maneiras; no exemplo em questão, os autores optam por uma análise qualitativa de conteúdo e fazem codificações e categorias de modo indutivo. Por fim, Häsel-Weide e Nührenbörger (2019) discutem sobre as diferenças entre as tarefas especificadas no livro e as atividades em sala de aula, mostrando que as atividades têm caráter mais aberto, levando a invenções autônomas por parte das crianças.

No trabalho de Shen, Bao e Zhang (2019) o foco está em realizar uma meta-análise das pesquisas recentes relacionadas à representação matemática em livros didáticos. Os procedimentos adotados pelos autores foram de meta-análise para sintetizar os resultados das pesquisas e, posteriormente, de análise de conteúdo para empregar inferências específicas nesses textos. A partir de uma base de dados internacional e outra chinesa, os autores chegam a 19 artigos que versam sobre representação matemática e livros didáticos.

Após leitura dos títulos e resumos, Shen et al. (2019) analisam seções específicas desses textos, como problema de pesquisa, conteúdo e métodos. Em sua grande maioria, as pesquisas têm um foco em livros do Ensino Fundamental (Anos Iniciais e Finais) e nos conteúdos de Aritmética, Álgebra e Geometria. Entre os métodos e os problemas de pesquisa destacam-se a análise de documentos e a pesquisa comparativa, além das descrições de características dos livros didáticos. Os autores, então, apontam a necessidade de ampliação dos métodos e das perguntas de pesquisa, além da importância de relacionar esses estudos com os alunos, professores e tecnologias.

Jahnke (2019), por sua vez, relata um projeto e o workshop desenvolvido por ele para ensinar a escrever livros didáticos de matemática. Além de considerar a importância desse recurso, o autor afirma que diversas mudanças ocorrem no cenário educacional, sendo necessária a escrita de novos livros para atender a essa demanda. Jahnke (2019) mostra os componentes teóricos e práticos do projeto, como palestras sobre o currículo, a escrita, a importância e os elementos dos livros didáticos assim como os problemas de matemática e os problemas de palavras (word problems). Os participantes do projeto desenvolveram textos diversos, como a escrita de jogos, contendo regras e procedimentos. O workshop durou cinco dias e contou com 20 participantes, entre eles professores, doutorandos e educadores. O autor traz alguns 
resultados com o objetivo de melhorar o projeto e leválo para países diferentes; as dificuldades por parte dos participantes foram em relação ao pouco tempo e pelo fato de não conhecerem seus leitores, para conversas e apontamentos presenciais, por exemplo.

O trabalho de Tarp (2019) conta com a utilização da abordagem funcional da linguagem (Halliday, 1973) para analisar as competências para "dominar muitos", contando e adicionando (Tarp, 2018). O autor evidencia ser possível a elaboração de um currículo para todos os alunos, sem a necessidade de dividi-lo em faixas e que o "domínio de muitos" que as crianças trazem para a escola contém a matemática básica como proporcionalidade, cálculo, resolução de equações e modelagem por sentenças numéricas que podem ser associadas a um nível de gramática, segundo a abordagem funcional da linguagem.

É apropriado para os alunos escolherem a face inferior como uma estratégia para imaginar tarefas de dobramento mental? E há um aumento no uso prático dos alunos dessa estratégia espacial? São as perguntas que direcionam o trabalho de Watanabe (2019). O modelo teórico adotado foi o proposto por Uttal et al. (2013) que identificam as operações mentais como um tipo de raciocínio espacial classificado como uma habilidade intrínseca-dinâmica. Para tanto, foram planejadas duas aulas segundo a metodologia lesson study para desenvolver as habilidades espaciais de 32 alunos da $4^{\text {a }}$ série usando o dobramento mental. Como resultado, Watanabe (2019) identificou que, a partir de um padrão, os alunos poderiam montar cubos mais difíceis e que a estratégia mental foi reconhecida como útil pelos alunos para resolver problemas semelhantes.

Como pode ser notado, essas duas últimas pesquisas não trazem discussões sobre o livro didático, mas, apesar disso, suas considerações sobre currículo (Tarp, 2019) e atividades de dobra mental (Watanabe, 2019) poderiam fazer parte de elementos que corroboram o que foi visto até aqui. Notamos que houve o predomínio de pesquisas descritivas e que os métodos foram diversificados como lesson study, design science, projeto e meta-análise, mostrando que as pesquisas com livros didáticos apresentam metodologias diferentes ao redor de um mesmo objeto de estudo. Em relação aos referenciais teóricos, percebemos, também, variedades nos trabalhos, como as questões políticas e sociais na Educação Matemática, as competências presentes no currículo, considerações filosóficas de modernização, práticas de inclusão e estudos sobre linguagem.

\subsection{Avaliação das novas ideias, métodos, resultados e conclusões}

A ICMT 3 nos traz algumas das perspectivas e direcionamentos mais atuais sobre os estudos a respeito do livro didático de matemática no âmbito da maioria dos continentes, contribuindo, assim, para o desenvolvimento e uma melhor compreensão desse campo de pesquisa. A Figura
1 ilustra a distribuição dos trabalhos, aqui apresentados, por continentes.

Figura 1 - Distribuição das pesquisas por continentes.

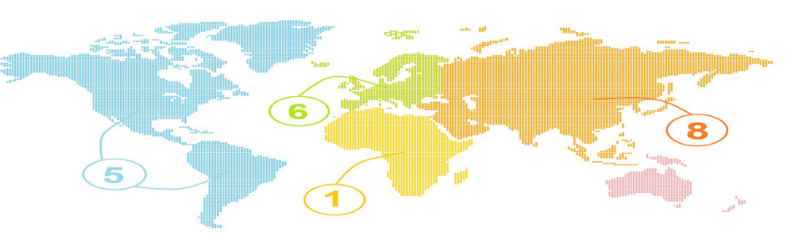

Fonte: Elaborado a partir do vetor criado por freepik.com.

A partir dos dados retratados na Figura 1, podemos observar que o continente asiático detém a maior quantidade de trabalhos. Constatamos que dos oito trabalhos apresentados nesse continente, cinco são japoneses. A Alemanha participa com quatro trabalhos dentre os seis estudos europeus. Não evidenciamos trabalhos provenientes da Oceania.

Nos estudos apresentados na referida conferência, a abordagem metodológica predominante pertence ao âmbito do paradigma da pesquisa qualitativa, com a utilização de vídeos, entrevistas, observação de aulas, mapeamento, análise de conteúdo e/ou a combinação de alguns desses métodos. A variedade de métodos empregados para a produção e análise de dados coaduna-se com o paradigma de pesquisa qualitativa em que os pesquisadores empregam "uma ampla variedade de práticas interpretativas interligadas, na esperança de sempre conseguirem compreender melhor o assunto que está ao seu alcance." (Denzin \& Lincoln, 2006, p. 17). Essa diversidade de métodos permite captar nuances do objeto em estudo, o que contribui para melhor compreendê-lo.

Diferentes perspectivas teóricas foram apresentadas na ICMT 3 e, aqui, resumimos algumas das tendências que foram usadas repetidamente como a ideia do tetraedro sociodidático e a Capacidade de Design Pedagógico. Rezat e Sträßer (2012), baseados na Teoria da Atividade de Yrjö Engeström, propõem o tetraedro sociodidático com o objetivo de entender o papel central do uso de recursos no ensino de matemática. $\mathrm{O}$ modelo proposto pelos autores amplia a ideia do tetraedro original cujos vértices são: professor, aluno, conteúdo matemático e artefato (nesse caso o livro didático) considerando os parâmetros sociais e institucionais do ensino e da aprendizagem que afetam a interação em sala de aula.

Rezat e Sträßer (2012) destacam que não pretendem ver o tetraedro como uma figura regular e nem mesmo dispor de métricas sobre ele, pois, "os segmentos entre os pontos indicam apenas relações, sem distâncias ou outras medidas." (p. 651). Assim, não temos uma "hierarquia" entre os vértices, pois apenas indicam as relações existentes entre eles. $\mathrm{Na}$ perspectiva de Mesa et al. (2019) o modelo possibilita que os pesquisadores percebam simultaneamente o uso dos recursos pelos alunos ao aprenderem matemática e o modo pelo qual professores e alunos interagem com os recursos. 
A Capacidade de Design Pedagógico é definida Brown (2009) como a "capacidade do professor para perceber e mobilizar os recursos existentes para criar episódios instrucionais", ou seja, "descreve a maneira e o grau em que os professores criam, intencionalmente, planejamentos produtivos que ajudam a atingir seus objetivos educacionais." (p. 29).

A relevância da Capacidade de Design Pedagógico para os estudos relacionados aos livros didáticos é, de acordo com o autor, orientar o entendimento sobre o que os professores trazem para suas interações com livros didáticos e outros recursos, sinalizando que a sua utilização em sala de aula envolve algumas habilidades na elaboração, no planejamento e na execução de sua ação educativa. Até porque, na visão do autor, a ideia da Capacidade de Design Pedagógico considera não apenas o que o professor sabe, mas sua capacidade de realizar novas atividades com esse conhecimento. A compreensão da Capacidade de Design Pedagógico permite explorar as capacidades dos professores para identificar e mobilizar recursos dos livros didáticos de forma eficaz.

No que tange ao uso dos livros didáticos, nessa conferência, o foco maior foi para as análises que se debruçaram para investigar o uso dos livros pelo aluno, contrariando o que fora apontado por Rezat (2011), ou seja, que a maior atenção dos pesquisadores era destinada para o uso do livro didático pelos professores. O dado encontrado no mapeamento aqui realizado indica certa mudança de foco nas pesquisas mais atuais.

A questão da interação entre os alunos e entre os alunos e o professor vem sendo apresentada com maior ênfase na categoria dos livros digitais. Além de explicar as diferenças para os livros impressos ou digitalizados (cópia digital do livro impresso), como a utilização de recursos que fazem parte dos livros digitais, os autores dessa categoria mostram a dinâmica em ambientes virtuais, uma vez que os alunos estão conectados e as discussões sobre habilidades matemáticas oportunizam uma reflexão sobre a aprendizagem desses alunos.

Em relação à categoria outros, destacamos que os trabalhos de Højgaard (2019) e Jahnke (2019) proporcionam visões diferentes em relação ao livro didático como objeto de estudo, uma vez que esses pesquisadores também são autores de livros. O projeto que Jahnke (2019) desenvolveu sobre a escrita de livros didáticos, nos mostra a preocupação do autor em relação ao desenvolvimento de livros em países diferentes como o Iêmen e Vietnã, além de perceber que mudanças são contínuas e os livros devem acompanhar esse movimento. Højgaard (2019), por sua vez, deixa claro que as competências curriculares são a base para a escrita de uma coleção dinamarquesa de livros didáticos de matemática, evidenciando a preocupação de alinhar a estrutura curricular com os objetivos de aprendizagem em cada capítulo. Assim, pontuamos que a escrita de livros didáticos se mostra como relevante para análises futuras de outros pesquisadores, bem como por apresentar a forma com que os autores se debruçam para esse desenvolvimento, observando quais são os elementos e as características essenciais para essa empreitada.

Alguns princípios para a escrita e para o desenvolvimento de livros didáticos são apresentados por Fan (2010). O autor lista seis princípios relevantes nessa tarefa, a saber: princípio do currículo, princípio da disciplina, princípio da pedagogia, princípio da tecnologia, princípio do contexto e princípio da apresentação. Posteriormente, Fan et al. (2013) indicam, como possibilidade para ampliar o campo da pesquisa sobre livro didático, questões relacionadas ao seu desenvolvimento e à sua produção.

\section{Conclusão}

Percebemos que o campo de pesquisas sobre livros didáticos de matemática está evoluindo, ampliando as vertentes teóricas e consolidando alguns dos métodos empregados para análise e produção dos dados. Notamos que os trabalhos aqui categorizados procedem de pesquisadores de alguns países em específico, mesmo em se tratando de um evento que contou com a participação de autores de todos os continentes, conforme apontado anteriormente.

Ponderamos que, em alguns dos trabalhos, não há uma descrição clara dos objetivos e dos referenciais teóricometodológicos, o que faz com que a compreensão dos relatos dessas pesquisas fique, de certa forma, prejudicada. Isso se deu, principalmente, com alguns dos estudos apresentados no formato de pôster, visto que o espaço era mais limitado, em comparação com as comunicações orais.

Diante do que percebemos, os focos temáticos sobre livros didáticos de matemática, que têm emergido nos estudos apresentados na terceira edição da ICMT, neste recorte aqui apresentado, são os livros digitais, o uso do livro didático, seja pelo aluno, pelo professor ou por ambos, além de trabalhos que problematizam a distribuição dos livros didáticos e a escrita desses recursos; os olhares filosóficos sobre os livros e as relações com práticas inclusivas, além de trabalho do tipo meta-análise. Salientamos que emergiu, também, a categoria análise do livro didático, seja ela comparativa, do conteúdo ou desenvolvida pelos pesquisadores e outros sujeitos, que será tema de outro texto.

Pontuamos dois direcionamentos para pesquisas futuras: a utilização dos livros digitais e o papel das políticas e contexto econômico brasileiro. Indicamos que a utilização de livros didáticos digitais, principalmente em decorrência do ensino remoto ocasionado pela pandemia da Covid-19, poderia ter contribuído para uma maior aproximação entre professor e alunos. Entendemos, ainda, como mostrado por Borba, Chiari e Almeida (2018), que a construção de um Material Didático Digital Interativo fornece a descrição de como a comunicação em ambientes virtuais de aprendizagem é transformada e registrada, automaticamente, nesse ambiente, gerando uma espécie de livro digital interativo. Assim, no lugar de usar os livros digitais em sala de aula presencial, como mostrado pelas 
pesquisas da categoria analisada, esses materiais poderiam fazer parte dos recursos utilizados pela turma em momentos de ensino remoto, contribuindo dessa maneira, para uma melhor interação entre o professor e os alunos.

Compreendemos que a categoria dos livros digitais pode ser vista como um avanço para as pesquisas que tratam o livro didático como objeto de estudo na Educação Matemática. Fan et al. (2013) pontuaram que as pesquisas com os livros eletrônicos poderiam ser um foco emergente e explorado pelos pesquisadores. Como as pesquisas com os livros digitais estavam em um estágio inicial à época, os autores citados incluíram os livros eletrônicos na categoria "outros" em seu levantamento e, em nossa pesquisa, percebemos que se trata de uma nova categoria. Apesar de os livros digitais emergirem como uma categoria nesse mapeamento, no contexto brasileiro ainda não foram evidenciadas pesquisas que tratam do tema, conforme levantamento das últimas três edições do Seminário Internacional de Pesquisa em Educação Matemática, realizado por Perovano e Ribeiro Guimarães (2020).

O trabalho de Andrade-Molina e Montecino (2019) apresenta uma discussão necessária em relação à compra, com dinheiro público, de livros didáticos produzidos por editoras privadas. Além de mostrar que os livros que chegam às escolas privadas e públicas são diferentes, os autores apresentam características neoliberais presentes nessa aquisição. Refletindo sobre o cenário brasileiro, em que o Programa Nacional do Livro e do Material Didático (PNLD) faz a avaliação, aquisição e distribuição dos livros para todos os alunos das escolas públicas (Mazzi, 2018), apontamos que essa discussão poderia ser ampliada para analisar o mercado produtor de livros brasileiros, conforme assinalam Santos e Silva (2019), quando debatem a constituição do livro de matemática como um produto comercial lucrativo.

Esperamos contribuir, no sentido de fomentar reflexões no que tange ao desenvolvimento da pesquisa sobre os livros didáticos de matemática ampliando, assim, esse campo de pesquisa. Ademais, para compreender a produção de conhecimento a respeito desse objeto de estudo implica a realização de um estado da arte, que inclua as pesquisas acadêmicas em teses e dissertações, além dos livros, capítulos de livros, periódicos e anais de outros eventos que fazem parte de uma série de trabalhos que vêm sendo desenvolvidos no âmbito do Grupo de Pesquisa teorEMa - Interlocuções entre Geometria e Educação Matemática, do qual os autores participam.

\section{Agradecimentos}

À Coordenação de Aperfeiçoamento de Pessoal de Nível Superior - Brasil (CAPES) - Código de Financiamento 001, pela bolsa de mestrado concedida ao primeiro autor. Agradecemos, também, aos integrantes do Grupo de Pesquisa teorEMa - Interlocuções entre Geometria e Educação Matemática que, embora não sejam responsáveis pelo conteúdo deste texto, contribuíram em algum momento de sua elaboração com comentários e críticas.

\section{Referências}

Andrade-Molina, M. \& Montecino, A. (2019). On the Marketing and Distribution of School Mathematics Textbooks in a Neoliberal Context. In: Rezat, S., Fan, L., Hattermann, M., Schumacher, J., \& Wuschke, H. (Eds.). Proceedings ... (p. 125-130).

Beezer, R. (2019). First course in linear algebra. Gig Harbour: Congruent Press.

Borba, M. C., Almeida, H. R. F. L. \& Gracias, T. A. S. (2018). Pesquisa em ensino e sala de aula: diferentes vozes em uma investigação. Belo Horizonte, MG: Autêntica.

Borba, M.C., Chiari, A.S.S. \& Almeida, H.R.F.L. (2018). Interactions in virtual learning environments: new roles for digital technology. Educational Studies in Mathematics, 98(3), 269-86. doi: https://doi.org/10.1007/s10649-0189812-9

Brnic, M. (2019). The Use of a Digital Textbook With Integrated Digital Tools. In: Rezat, S., Fan, L., Hattermann, M., Schumacher, J., \& Wuschke, H. (Eds.). Proceedings ... (p. 369-370).

Brown, M.W. (2009). The teacher-tool relationship: Theorizing the design and use of curriculum materials. In J. T. Remillard, B. A. Herbel-Eisenmann \& G. M. Lloyd. Mathematics teachers at work: Connecting curriculum materials and classroom instruction (p.17-36). New York: Routledge.

De Almeida, L. M. W. \& Silva, K. A. P. (2019). The Learning of Double Integral Concept Using a Textbook. In: Rezat, S., Fan, L., Hattermann, M., Schumacher, J., \& Wuschke, H. (Eds.). Proceedings ... (p. 149-154).

Denzin, N.K. \& Lincoln, Y.S. (2006). O planejamento da pesquisa qualitativa: teorias e abordagens. Porto Alegre: Artmed.

Dörfler, W. (1991). Forms and means of generalization in mathematics. In A. J. Bishop, S. Mellin-Olsen \& J. v. Dormolen. Mathematical knowledge: Its growth through teaching (p.63-85). Dordrecht: Kluwer.

Edson, A. J., Phillips, E. D. \& Bieda, K. (2019). Transitioning From Print to Digital Curriculum Materials: Promoting Mathematical Engagement and Learning. In: Rezat, S., Fan, L., Hattermann, M., Schumacher, J., \& Wuschke, H. (Eds.). Proceedings ... (p. 166-172).

Fan, L. (2010). Principles and processes for publishing textbooks and alignment with standards: A case in Singapore. In APEC Conference on Replicating Exemplary Practices in Mathematics Education, Koh Samui, Thailand (p. 7-12).

Fan, L., Hattermann, M., Schumacher, J., \& Wuschke, H. (Eds.). Proceedings ... (p. 197-202).

Fan, L., Zhu, Y. \& Miao, Z. (2013). Textbook research in mathematics education: development status and directions. ZDM Mathematics Education, 45(5), 633-46. doi: https://doi. org/10.1007/s11858-013-0539-x

Ferreira, N. S. D. A. (2002). As pesquisas denominadas "estado da arte". Educação \& Sociedade, 23(79), 257-72. doi: https:// doi.org/10.1590/S0101-73302002000300013

Fiorentini, D., Grando, R. C., Miskulin, R. G. S., Crecci, V. M., Lima, R. D. \& Costa, M. C. (2016). O professor que ensina matemática como campo de estudo: concepção do projeto de pesquisa. In D. Fiorentini, C. L. B. Passos, \& R.C.R. Lima. Mapeamento da pesquisa acadêmica brasileira sobre o professor que ensina matemática: periodo, 2001-2012, (p. 


\section{7-42). Campinas: FE/UNICAMP.}

Gitirana, V., Miyakawa, T., Rafalska, M., Soury-Lavergne, S. \& Trouche, L. (2018). Proceedings of the Re(s)sources 2018 International Conference.

Glasnović Gracin, D. \& Jukić Matić, L. (2019). Same Textbook, Different Points Of View: Students and Teachers as Textbook Users. In: Rezat, S., Fan, L., Hattermann, M., Schumacher, J., \& Wuschke, H. (Eds.). Proceedings ... (p. 173-178).

Goto, M. (2019). Current Status and Issues of Digital Teaching Materials in Japan. In: Rezat, S., Fan, L., Hattermann, M., Schumacher, J., \& Wuschke, H. (Eds.). Proceedings ... (p. 371-372).

Halliday, M. (1973). Explorations in the function of language. London: Edward Arnold.

Häsel-Weide, U. \& Nührenbörger, M. (2019). Materials for Inclusive Mathematics Education-Design Principles and Practices. In: Rezat, S., Fan, L., Hattermann, M., Schumacher, J., \& Wuschke, H. (Eds.). Proceedings ... (p. 185-190).

Højgaard, T. (2019). Competencies and Textbook Development: a Three-dimensional Content Model Enacted in the Danish Textbook Series Matematrix For Grades K-9. In: Rezat, S.,

Jahnke, T. (2019). Report on the Design and Realisations of the Workshop 'Writing Maths Textbooks. In: Rezat, L., Fan, M., Hattermann, J., Schumacher, \& H., Wuschke. Proceedings ... (p. 203-208).

Kelly, G.A. (1955). The psychology of personal constructs. New York: Norton.

Korsunsky, B. (2002). Improper Use of physics-related context in high school mathematics problems: Implications for learning and teaching. School Science and Mathematics, 102(3), 10713. doi: https://doi.org/10.1111/j.1949-8594.2002.tb17904.x

Lemaire, P. \& Siegler, R.S. (1995). Four aspects of strategic change: contributions to children's learning of multiplication. Journal of Experimental Psychology: General, 124(1), 83-96. doi: https://psycnet.apa.org/doi/10.1037/0096-3445.124.1.83

Leshota, M. (2019). Understanding Mathematics Teachers' Pedagogical Design Capacity in Contexts of High Prescription. In: Rezat, L., Fan, M., Hattermann, J., Schumacher, \& H., Wuschke. Proceedings ... (p. 221-226).

Mabuchi, T. \& Hayata, T. (2019). A Study on the Educational Ideology of Modernization in the Case of Japanese Textbook. In: ., Rezat, L., Fan, M., Hattermann, J., Schumacher, \& H., Wuschke. Proceedings ... (p. 377-378).

Mazzi, L.C. (2018). As demonstrações matemáticas presentificadas nos livros didáticos do Ensino Médio: um foco nos capitulos de Geometria. Tese de doutorado, Programa de Pós-Graduação Multiunidades em Ensino de Ciências e Matemática, Instituto de Física "Gleb Wataghin", Universidade Estadual de Campinas. Campinas, Brasil.

Mesa, V. Liakos, Y. \& Zhang, H. (2019). Textbook Content in use: Manual and Machine Coding. In: Rezat, L., Fan, M., Hattermann, J., Schumacher, \& H., Wuschke. Proceedings ... (p. 251-256).

Mizoguchi, T. \& Shinno, Y. (2019). How Japanese Teachers use Mathematics Textbooks for "Kyozai-kenkyu": Characterizing their Different uses by Paradidactic Praxeologies. In: Rezat, S., Fan, L., Hattermann, M., Schumacher, J., \& Wuschke, H. (Eds.). Proceedings ... (p. 257-262).

Naftaliev, E. (2018). Prospective teachers' interactions with interactive diagrams: Semiotic tools, challenges and welltrodden paths. In L. Fan, L. Trouche, C. Qi, S. Rezat,
J. Visnovska. Research on Mathematics Textbooks and Teachers'Resources (p. 297-314). Springer. doi: https://doi. org/10.1007/978-3-319-73253-4_14

Nührenbörger, M., Rösken-Winter, B., Fung, C. I., Schwarzkopf, R., Wittmann, E.C., Akinwunmi, K., Lensing, F. \& Schacht, F. (2016). Design Science and its importance in the German mathematics educational discussion. Rotterdam: Springer. doi: 10.1007/978-3-319-43542-8

Pepin, B., Gueudet, G., Yerushalmy, M., Trouche, L. \& Chazan, D. (2015). E-textbooks in/for teaching and learning mathematics: A disruptive and potentially transformative educational technology. In L. English \& D. Kirshner. Handbook of international research in mathematics education (p. 636-61). New York: Taylor \& Francis.

Perovano, A. P. \& Ribeiro Guimarães, D. (2020). Um mosaico dos trabalhos publicados nos anais do SIPEM: foco nos livros didáticos e nos materiais curriculares. Intermaths: Revista de Matemática Aplicada e Interdisciplinar, 1(1), 34-51. doi: https://doi.org/10.22481/intermaths.v1i1.7395

Pollak, H. O. (1968). On some of the problems of teaching applications of mathematics. Educational Studies in Mathematics, 1(1), 24-30. doi: https://doi.org/10.1007/ BF00426228 and students' use of mathematics textbooks. In G. Gueudet, B. Pepin \& L. Trouche. From text to 'lived' resources: Mathematics curriculum materials and teacher development (p. 231-46). New York: Springer.

Rezat, S. \& Sträßer, R. (2012). From the didactical triangle to the socio-didactical tetrahedron: Artifacts as fundamental constituents of the didactical situation. ZDM Mathematics Education, 44(5), 641-651. doi: https://doi.org/10.1007/ s11858-012-0448-4

Rezat, S., Fan, L., Hattermann, M., Schumacher, J. \& Wuschke, H. (Eds.). (2019). Proceedings of the Third International Conference on Mathematics Textbook Research and Development. Paderborn, Germany. Paderborn: Universitätsbibliothek Paderborn. doi: 10.17619/ $\mathrm{UNIPB} / 1-768$.

Santos, J. W. \& Silva, M. A. (2019). Relações de poder na idealização de livros didáticos de Matemática. Práxis Educativa, 14(1), 250-272. doi: https://doi.org/10.5212/ PraxEduc.v.14n1.014

Schorcht, S. (2019). Histos - Teachers Connotations about Historical Snippets in Textbooks. In: ., Rezat, L., Fan, M., Hattermann, J., Schumacher, \& H., Wuschke. Proceedings ... (p. 299-304).

Shen, Y., Bao, J. \& Zhang, J. (2019). Research Actuality and Trend on the Mathematical Representations of Textbooks. In: Rezat, S., Fan, L., Hattermann, M., Schumacher, J., \& Wuschke, H. (Eds.). Proceedings ... (p. 305-310).

Sievert, H., Van den Ham, A. K. \& Heinze, A. (2019). Why the Textbook Matters: a Two-domain Impact Analysis. In: Rezat, S., Fan, L., Hattermann, M., Schumacher, J., \& Wuschke, H. (Eds.). Proceedings ... (p. 311-316).

Slisko, J., Hernández Rebollar, L.A., Nabor Sánchez, A. \& Ramirez Herrera, S. (2019). Inadequate Learning Sequence and Erroneous Fact-like Statement in a Mathematics Textbook: What Can Students Take From Them? In: S., Rezat, L., Fan, M., Hattermann, J., Schumacher, \& H., Wuschke. Proceedings ... (p. 317-323).

https://doi.org/10.26711/007577152790023

Tanaka, K. \& Hayata, T. (2019). A Study on the Senior High 
School Students' Algebraic Activities and the Digital Tools in Japanese Digital Mathematics Textbook. In: S., Rezat, L., Fan, M., Hattermann, J., Schumacher, \& H., Wuschke. Proceedings ... (p. 387-8).

Tarp, A. (2018). Mastering many by counting, re-counting and double-counting before adding on-top and next-to. Journal of Mathematics Education, 11(1), 103-117. doi:

Tarp, A. (2019). Developing the Child's Own Mastery of Many. In: Rezat, S., Fan, L., Hattermann, M., Schumacher, J., \& Wuschke, H. (Eds.). Proceedings ... (p. 329-334).
Uttal, D. H., Meadow, N. G., Tipton, E., Hand, L. L., Alden, A. R., Warren, C. \& Newcombe, N. S. (2013). The malleability of spatial skills: Mind, cognitive science, and human experience. Cambridge, MA: MIT Press.

Valente, W. R. (2008). Livro didático e educação matemática: uma história inseparável. Zetetiké, 16(30), 139-162. doi: https://doi.org/10.20396/zet.v16i30.8646894

Watanabe, S. (2019). A Lesson Study Intervention to Develop Primary School Students' Ability to Perform Mental Folding Operations'. In: S., Rezat, L., Fan, M., Hattermann, J., Schumacher, \& H.,Wuschke. Proceedings ... (p. 347-352). 